Clinical implications of infection with a novel metastrongyloid species in the red panda (Ailurus fulgens)

Willesen, Jakob; Meyland-Smith, Frederik; Wiinberg, Bo; Monrad, Jesper; Bertelsen, Mads

Published in:

Journal of Zoo and Wildlife Medicine

DOI:

$10.1638 / 2011-0100.1$

Publication date:

2012

Document version

Early version, also known as pre-print

Citation for published version (APA):

Willesen, J., Meyland-Smith, F., Wiinberg, B., Monrad, J., \& Bertelsen, M. (2012). Clinical implications of infection with a novel metastrongyloid species in the red panda (Ailurus fulgens). Journal of Zoo and Wildlife Medicine, 43(2), 283-288. https://doi.org/10.1638/2011-0100.1 


\title{
CLINICAL IMPLICATIONS OF INFECTION WITH A NOVEL METASTRONGYLOID SPECIES IN THE RED PANDA (AILURUS FULGENS)
}

\author{
Jakob L. Willesen, D.V.M., Ph.D., Frederik Meyland-Smith, D.V.M., Bo Wiinberg, D.V.M., Ph.D., \\ Jesper Monrad, D.V.M., Ph.D., Dipl. E.V.P.C., and Mads F. Bertelsen, D.V.M., D.V.Sc., Dipl. \\ A.C.Z.M.
}

\begin{abstract}
In a recent survey, $30 \%$ of the European red panda (Ailurus fulgens) population was found to be infected with a newly discovered metastrongyloid nematode. In a following prospective study, four naturally infected captive-bred red pandas infected with this parasite were examined and compared with two uninfected control animals. On clinical examination, no abnormalities were detected with respect to vital parameters and cardiovascular system in all six examined animals. Similarly, few and nonspecific changes were recorded on serum biochemistry. No changes on pulmonary pattern were noted on thoracic radiographs. Vertebral heart scores were between 7.2 to 8.6 , and no difference was noted between infected and control animals. Two animals had slightly prolonged clotting time and reaction time on thromboelastography but not likely to be of clinical relevance. In conclusion, infection with the newly identified metastrongyloid nematode in the red pandas seems to have little or no clinical importance.
\end{abstract}

Key words: Ailurus fulgens, metastrongyloid, clinical signs, infection, nematode, red panda.

\section{INTRODUCTION}

The red panda (Ailurus fulgens) is a herbivorous carnivore ${ }^{16}$ originating from subtropical and temperate forests of eastern Asia. ${ }^{6,17}$ The species has been kept in European zoos since 1869, ${ }^{12}$ and the current populations of 212 animals in 91 zoos are all captive bred. ${ }^{8}$ Angiostrongylus vasorum is a metastrongyloid parasite inhabiting the pulmonary arteries and right side of the heart of domestic canids throughout Europe and other parts of the world. ${ }^{3}$ It infects canids and related species such as fox (Vulpes vulpes), wolf (Canis lupus), coyote (Canis latrans), jackal (Canis aureus), European otter (Lutra lutra), ferret (Mustela putorius), and badger (Meles meles) ${ }^{14}$ as well as in red pandas kept in captivity. ${ }^{9} 15$ Clinical signs originating from the respiratory tract such as cough and exercise intolerance as a consequence

From the Department of Small Animal Clinical Sciences, Faculty of Health and Medical Sciences, University of Copenhagen, Dyrlaegevej 16, DK-1870 Frederiksberg C, Denmark (Willesen, Wiinberg); Center for Zoo and Wild Animal Health, Copenhagen Zoo, Roskildevej 38, DK-2000 Frederiksberg, Denmark (Meyland-Smith, Bertelsen); and Danish Centre for Experimental Parasitology, Faculty of Health and Medical Sciences, University of Copenhagen, Dyrlaegevej 100, DK-1870 Frederiksberg C, Denmark (Monrad). Present address (Meyland-Smith): Skovlunde-Herlev Veterinary Clinic, Skovlunde Byvej 53, DK-2740 Skovlunde, Denmark. Correspondence should be directed to Dr. Bertelsen (mfb@zoo.dk). of verminous pneumonia are the clinical manifestations most often related to $A$. vasorum infections in dogs. Severe complications such as bleeding disorders and even death may ensue. Due to the morphologic similarities of this novel metastrongyloid first-stage $\left(\mathrm{L}_{1}\right)$ larvae compared with the $\mathrm{L}_{1}$ larvae of $A$. vasorum, one focus of the present study was on the cardio-respiratory system, because a similar life cycle and therefore similar clinical manifestations could be suspected in the two parasites. Deaths etiologically attributed to infections with $A$. vasorum have been reported in a total of five cases in red pandas in European zoos. ${ }^{9,11,15}$ One animal died with no premonitory signs, but the remaining animals died after treatment or were euthanized. Abnormal breathing and weight loss were the clinical signs observed in a male red panda in Bristol Zoo Gardens in 2006. ${ }^{15}$ Lesions found on necropsy were described as verminous pneumonia with different degrees of mineralization and fibrosis. In a recent study, 115 red pandas originating from 54 European zoos were coprologically examined for excretion of metastrongyloid $L_{1}$ nematode larvae by using the Baermann method. ${ }^{1}$ In the same study, 3 of 115 red pandas (2.6\%) were found to be infected with $A$. vasorum and 32 of 115 red pandas $(27.8 \%)$ excreted metastrongyloid $\mathrm{L}_{1}$ of a previously undescribed morphologic type. ${ }^{1}$ None of the 32 animals diagnosed with this new metastrongyloid nematode displayed obvious clinical symptoms. Clinical and paraclinical examinations such as clinical chemistry or thoracic 
radiographs were not performed in any of these cases, and exact knowledge of their clinical status therefore was lacking. The life cycle of this novel metastrongyloid nematode species and its migration route within the red panda have not been described previously. The purpose of the present study was to prospectively investigate the potential clinical and paraclinical impacts of the novel metastrongyloid nematode infection in red pandas.

\section{MATERIALS AND METHODS}

\section{Infected group}

The infected group consisted of four red pandas. All of these animals were housed in Aalborg Zoo, Denmark. The first panda (P1) was a sexually mature female, $4.5 \mathrm{yr}$ of age, bred in Italy, and imported to Aalborg Zoo 3 yr earlier. The second panda (P2) was a sexually mature male, $8.5 \mathrm{yr}$ of age, bred in France, and imported to Aalborg from Germany $5 \mathrm{yr}$ earlier. The third and fourth pandas (P3 and P4) were both female cubs, 6 mo old, bred in Aalborg Zoo and offspring of P1 and P2. All four red pandas were kept together in the same enclosure and were diagnosed as being infected with the metastrongyloid nematode by using a modified Baermann method for coprologic examination and morphologic identification. ${ }^{1}$ During sampling periods, the animals were separated. None of the animals displayed clinical signs such as coughing or exercise intolerance before coprologic examinations. The infected animals had not received any anthelmintic treatment for the past 18 mo before sampling.

\section{Control group}

The control animals were housed in the Copenhagen Zoo, Denmark. The group consisted of one 6-yr-old male (C1) bred in the United Kingdom and imported $3 \mathrm{yr}$ earlier, and one 11-mo-old female (C2) born in France and imported 5 mo before the study. The group was selected as controls based on consistently negative Baermann tests, and routine prophylactic anthelmintic treatment with milbemycin (Interceptor ${ }^{\circledR}$ Flavor tabs, Novartis Animal Health US, Inc., Greensboro, North Carolina 27408, USA; 2.5 mg/animal p.o., q90d).

\section{Examinations}

For clinical examinations, the animals were anesthetized using a combination of medetomidine (Cepetor, CP-Pharma, D-31303 Burgdorf,
Germany; mean, $0.06 \mathrm{mg} / \mathrm{kg}$ i.m.), midazolam (Dormicum, Roche A/S, 2650 Hvidovre, Denmark; mean, $0.3 \mathrm{mg} / \mathrm{kg}$ i.m.), and ketamine (Ketaminol, Intervet International B.V., 5831 AN Boxmeer, Holland; mean, $4.2 \mathrm{mg} / \mathrm{kg}$ i.m.). All the animals received oxygen by mask while anesthetized. Some individuals (P1, P2, P4, and C1) were supplemented with isoflurane (Isoba, Schering-Plough Animal Health, 2750 Ballerup, Denmark) by facemask to maintain anesthesia during the procedures. A thorough clinical examination was performed, with emphasis on the body condition of the animals; auscultation of the heart and lungs; body temperature, and assessment of buccal mucosal membranes; and skin and eyes for evidence of bleeding abnormalities. Thoracic radiographs in both left and right lateral and ventrodorsal positioning were obtained during maximal inspiration and subsequently evaluated for pulmonary changes and evidence of an enlarged heart by recording of vertebral heart size (VHS). ${ }^{4}$ All assessments of radiographic changes were performed by the same trained radiologist by using a standardized examination protocol.

For blood samples, $10 \mathrm{ml}$ of blood in total was obtained by the jugular vein, and blood analysis included hematologic, biochemical, and coagulation parameters as well as thromboelastography (TEG). Blood samples were collected into citrate, plain, and EDTA-treated Vacuette containers (Greiner Bio-One, 4550 Kremsmuenster, Austria). Serum biochemistry profiles were determined using an automated spectrophotometric analyzer (ADVIA1650, Siemens AG, $80333 \mathrm{Mu}-$ nich, Germany). Hematological profiles were determined using an automated hematologic analyzer (ADVIA 120, Siemens AG), with subsequent morphologic evaluation. ${ }^{13}$ Coagulation tests were performed on citrate-stabilized plasma using an automated analyzer (ACL Top 500, ILS Laboratories Scandinavia, DK-3450 Allerød, Denmark) that measured activated partial thromboplastin time (APTT), plasma fibrinogen, and prothrombin time (PT). Citrate-stabilized plasma also was used for thromboelastography (Hemostasis Analyzer 5000, Haemonetics Corporation, Braintree, Massachusetts 02184, USA). TEG analysis was conducted using recombinant human tissue factor (TF). ${ }^{18}$ All analyzers were subjected to daily internal quality control and quarterly external quality control measures. Only test results originating from accepted analytical runs were included. TEG was performed on site, 30 min after sampling, whereas all other blood analyses was performed the following day by the 
Table 1. General information and clinical examination results of four red pandas (P1-P4) infected with a newly discovered metastrongyloid nematode and two healthy controls (C1 and $\mathrm{C} 2)$.

\begin{tabular}{lcccccc}
\hline & P1 & P2 & P3 & P4 & C1 & C2 \\
\hline Sex & Female & Male & Female & Female & Male & Female \\
Weight $(\mathrm{kg})$ & 5.8 & 6.0 & 3.8 & 4.1 & 7.2 & 4.5 \\
Body condition & Good & Good & Good & Good & Good & Good \\
Age & 4 yr 5 mo & 8 yr, 5 mo & 5 mo & 5 mo & 6 yr & 11 mo \\
Temp. $\left({ }^{\circ} \mathrm{C}\right)$ & 38.8 & 37.4 & 38.0 & 38.8 & 38.0 & 37.9 \\
Mucous membranes & NAD & NAD & NAD & NAD & NAD & NAD \\
Heart rate $($ bpm) & 96 & 116 & 104 & 108 & 120 & 96 \\
Auscultation heart & Normal & Normal & Normal & Normal & Normal & Normal \\
Auscultation lungs & Normal & Normal & Normal & Normal & Normal & Normal \\
\hline
\end{tabular}

${ }^{a}$ Beats per minute.

Central Laboratory, Department of Small Animal Clinical Sciences, Faculty of Health and Medical Sciences, University of Copenhagen, Denmark. Due to the small number of animals in the study groups, no statistical analysis was performed.

\section{RESULTS}

No abnormal findings were observed during the clinical examination of any of the animals in either group (Table 1). All pandas were in good body condition and vital parameters (body temperature, heart rate, and respiration rate) were within normal limits. Examination of mucosal membranes, skin, and eyes revealed no sign of bleeding abnormalities in any of the six animals investigated (Table 1).

Upon assessment of the thoracic radiographs, no abnormalities in pulmonary patterns in any of the animals on either lateral or ventrodorsal images were detected. The VHS was measured for each of the animals and varied between 7.2 and 8.6, with no apparent difference between the infected and control animals (Table 2).

Hematologic parameters, including eosinophil, neutrophil, and platelet counts, for all four infected animals as well as the uninfected controls were within reference ranges (Table 3 ).$^{10}$ For the biochemical profiles, the four infected animals had slightly elevated amylase values, whereas the controls were within reference values. No reference values exist for serum fructosamine in red pandas, but a slightly lower values were detected in the infected animals $(288-319 \mu \mathrm{mol} / \mathrm{L})$ compared with the controls (399-427 $\mu \mathrm{mol} / \mathrm{L})$. All other parameters were within normal limits in both groups (Table 3 ).

The results of coagulation analyses revealed no apparent difference between groups for APTT, $\mathrm{PT}$, and plasma fibrinogen, with no reference values available (Table 4). Similarly, no difference existed between the infected and the control group in the TF-activated TEG parameters reaction time (R), clotting time (K), angle $(\alpha)$, and maximal clotting strength (MA). However, for lysis at $30 \mathrm{~min}$ (LY30), two of the infected animals (P2 and P3) had values of 46.8 and $27.5 \%$ compared with a range from 6.0 to $8.2 \%$ in the control group. For LY60, two of the infected animals (P2 and P3) had values of $45.5-54.0 \%$, whereas the range in the control group was 10.1$10.2 \%$ (Table 4 ).

\section{DISCUSSION}

The four pandas infected with the novel metastrongyloid nematode showed no clinical signs compared with the two animals in the control group based on a physical examination. Furthermore, very few and nonspecific changes were recorded on the performed paraclinical examinations. None of the infected animals showed any clinical signs before investigation, and all were in good body condition, with no detectable clinical changes on physical examination. In previous red pandas cases attributed to $A$. vasorum infection, thoracic radiographs showed a diffuse bronchiointerstitial pulmonary pattern throughout all

Table 2. Cardiac parameters and vertebral heart size of four red pandas (P1-P4) infected with a newly discovered metastrongyloid nematode and two healthy controls ( $\mathrm{C} 1$ and $\mathrm{C} 2)$ measured and calculated according to Buchanan and Bunnell. ${ }^{4}$

\begin{tabular}{lllllll}
\hline & P1 & P2 & P3 & P4 & C1 & C2 \\
\hline $\mathrm{L}^{\mathrm{a}}$ & 4.1 & 4.9 & 4 & 4.3 & 4.1 & 4.0 \\
$\mathrm{~W}^{\mathrm{b}}$ & 4.5 & 3.5 & 3.2 & 3.8 & 3.2 & 3.5 \\
VHS $^{\mathrm{c}}$ & 8.6 & 8.4 & 7.2 & 8.1 & 7.3 & 7.5 \\
\hline
\end{tabular}

${ }^{a}$ Long axis dimensions.

${ }^{\mathrm{b}}$ Short axis dimensions.

'VHS, vertebral heart size. 
Table 3. Hematologic and serum chemistry parameters for four red pandas (P1-P4) infected with a newly discovered metastrongyloid nematode and two healthy controls (C1 and $\mathrm{C} 2)$.

\begin{tabular}{|c|c|c|c|c|c|c|c|}
\hline Parameter & $\mathrm{P} 1$ & P2 & P3 & $\mathrm{P} 4$ & $\mathrm{C} 1$ & $\mathrm{C} 2$ & Reference range $^{a}$ \\
\hline $\mathrm{WBC}\left(\times 10^{9} / \mathrm{L}\right)$ & 5.4 & 8.8 & 5.5 & 7.0 & 6.3 & 5.5 & $2.2-20.2$ \\
\hline Bands $\left(\times 10^{9} / \mathrm{L}\right)$ & 3.38 & 3.62 & 3.51 & 4.5 & 2.4 & 2.4 & $0.04-18.2$ \\
\hline Lymphocytes $\left(\times 10^{9} / \mathrm{L}\right)$ & 1.8 & 5.0 & 1.7 & 2.3 & 3.6 & 2.9 & $0.05-16.2$ \\
\hline Monocytes $\left(\times 10^{9} / \mathrm{L}\right)$ & 0.11 & 0.09 & 0.11 & 0.14 & 0.06 & 0.06 & $0-2.3$ \\
\hline Eosinophils $\left(\times 10^{9} / \mathrm{L}\right)$ & 0.11 & 0.09 & 0.16 & 0.07 & 0.18 & 0.09 & $0-2.3$ \\
\hline Basophils $\left(\times 10^{9} / \mathrm{L}\right)$ & 0 & 0 & 0 & 0 & 0.02 & 0.01 & $0-0.6$ \\
\hline RBC Count $\left(\times 10^{12} / \mathrm{L}\right)$ & 6.4 & 7.8 & 8.1 & 7.9 & 7.0 & 7.9 & $5.2-13.0$ \\
\hline Hemoglobin $(\mathrm{mmol} / \mathrm{L})$ & 5.4 & 7.0 & 7.0 & 7.0 & 6.0 & 7.0 & $4.3-10.3$ \\
\hline Hematocrit $(\mathrm{L} / \mathrm{L})$ & 0.27 & 0.34 & 0.35 & 0.33 & 0.29 & 0.34 & $0.25-0.6$ \\
\hline Platelet $\left(\times 10^{9} / \mathrm{L}\right)$ & 493 & 422 & 464 & 340 & 670 & 977 & $207-1,258$ \\
\hline $\operatorname{ALT}^{\mathrm{b}}(\mathrm{U} / \mathrm{L})$ & 41 & 84 & 32 & 68 & 35 & 70 & $7-452$ \\
\hline $\operatorname{ALP}^{\mathrm{c}}(\mathrm{U} / \mathrm{L})$ & 28 & 20 & 94 & 98 & 25 & 42 & $4-163$ \\
\hline $\operatorname{GGT}^{\mathrm{d}}(\mathrm{U} / \mathrm{L})$ & 1 & 3 & 0 & 4 & 1 & 3 & $0-15$ \\
\hline Glucose $(\mathrm{mmol} / \mathrm{L})$ & 19.6 & 15.6 & 10.5 & 11.2 & 10.0 & 8.1 & $2.3-17.7$ \\
\hline $\mathrm{BUN}^{\mathrm{e}}(\mathrm{mmol} / \mathrm{L})$ & 13.8 & 10.9 & 10.5 & 8.9 & 7.2 & 7.3 & $3.2-25.7$ \\
\hline Creatinine $(\mu \mathrm{mol} / \mathrm{L})$ & 91 & 64 & 47 & 51 & 70 & 61 & $35-168$ \\
\hline Amylase (U/L) & 766 & 795 & 697 & 907 & 497 & 509 & $0-644$ \\
\hline Lipase (U/L) & 38.2 & 50.2 & 28.2 & 26.4 & 47.2 & 26.0 & $5.8-184.9$ \\
\hline Cholesterol (mmol/L) & 3.5 & 4.0 & 4.4 & 5.0 & 4.9 & 6.1 & $0-9.4$ \\
\hline Bilirubin $(\mu \mathrm{mol} / \mathrm{L})$ & 0.1 & 0.2 & 0 & 0.2 & 0.5 & 0.7 & $0-19$ \\
\hline Albumin $(g / L)$ & 25.8 & 33.8 & 34.3 & 35.5 & 28.8 & 34.7 & $22-49$ \\
\hline Serum protein $(\mathrm{g} / \mathrm{L})$ & 45.8 & 65.8 & 49.4 & 58.8 & 52.6 & 62.2 & $49-91$ \\
\hline Fructosamine $(\mu \mathrm{mol} / \mathrm{L})$ & 288 & 319 & 311 & 296 & 399 & 427 & $\mathrm{NA}^{\mathrm{f}}$ \\
\hline Bile acid $(\mu \mathrm{mol} / \mathrm{L})$ & 3 & 1 & 2 & 2 & 7 & 2 & NA \\
\hline Calcium (mmol/L) & 2.0 & 2.1 & 2.5 & 2.5 & 2.0 & 2.2 & $1.7-3.0$ \\
\hline Magnesium (mmol/L) & 1.2 & 1.1 & 1.1 & 1.1 & 1 & 1.1 & $0.7-1.3$ \\
\hline Phosphate (mmol/L) & 2.2 & 1.6 & 2.6 & 2.7 & 1.3 & 1.8 & $0.7-2.8$ \\
\hline Sodium $(\mathrm{mmol} / \mathrm{L})$ & 130.4 & 131.6 & 133.0 & 135.1 & 132.6 & 133.0 & $121-148$ \\
\hline Potassium (mmol/L) & 5.4 & 4.9 & 4.5 & 4.3 & 4.9 & 5.2 & $2.4-8.1$ \\
\hline Globulin $(\mathrm{g} / \mathrm{L})$ & 20 & 32 & 15.1 & 23.3 & 23.8 & 27.5 & $16-63$ \\
\hline
\end{tabular}

${ }^{a} \mathrm{U} / \mathrm{L}$ indicates units per liter. Reference ranges according to ISIS. ${ }^{10}$

b ALT, alanine aminotransferase, units per liter.

c ALP, alkaline phosphatase, units per liter.

${ }^{d}$ GGT, $\gamma$-glutamyltransferase, units per liter.

${ }^{\circ}$ BUN, blood urea nitrogen.

${ }^{\mathrm{f}} \mathrm{NA}$, not available.

lobes. Patchy alveolar filling and foci of mineralization also were present. In dogs and foxes experimentally infected with $A$. vasorum, radiographic changes of bronchial and interstitial as well as alveolar pulmonary patterns have been described previously. Similar radiographic changes have been described in naturally infected dogs. ${ }^{2}$ Here, no changes to the thoracic radiographs were recorded in the infected animals or in the control animals. A few reasons might explain this lack of change on radiographs. It is possible that this novel metastrongyloid is better adapted to the red pandas as a host and therefore is less pathogenic than $A$. vasorum to the host. The infected animals may have had very low-grade infections, causing changes too subtle to be detected by radiographs, or perhaps the lungs are not involved in the life cycle of this parasite to the same extent as in $A$. vasorum infections. If this was to be a highly pathogenic parasitic infection in the red panda, some degree of change to the radiographs would be expected in the four infected red pandas in this study. That $27.8 \%$ of the red pandas in European zoos were found infected with this novel nematode without reports of clinically affected animals may support the hypothesis that it is an infection of little or no pathogenic impact on its host. The recordings of VHS also showed no difference between the infected animals and the controls. This is in accordance with the normal thoracic radiographs, because right-sided cardiac enlargement secondary to pulmonary parenchymal disease usually requires significant changes for this to occur. Such extensive changes would have been 
Table 4. Coagulation and thromboelastograph parameters of four red pandas (P1-P4) infected with a newly discovered metastrongyloid nematode and two healthy controls ( $\mathrm{C} 1$ and $\mathrm{C} 2$ ). No reference range available.

\begin{tabular}{lrrrrrr}
\hline & P1 & P2 & P3 & P4 & C1 & C2 \\
\hline APTT $^{\mathrm{a}}(\mathrm{sec})$ & 15.0 & 14.8 & 15.6 & 14.8 & 15.4 & 15.0 \\
Fibrinogen (mg/dL) & 1.8 & 2.6 & 2.4 & 3.1 & 2.4 & 2.5 \\
PT $^{\mathrm{b}}(\mathrm{sec})$ & 8.1 & 7.7 & 8.4 & 7.0 & 8.6 & 7.2 \\
R $^{\mathrm{c}}(\mathrm{min})$ & 5.7 & 5.2 & 5.1 & 4.9 & 3.2 & 4.2 \\
K $^{\mathrm{d}}(\mathrm{min})$ & 1.6 & 1.2 & 1.7 & 1.0 & 0.8 & 0.8 \\
$\alpha^{\mathrm{e}}\left({ }^{\circ}\right)$ & 67.6 & 72.3 & 66.4 & 75.4 & 79.5 & 77.1 \\
MA $^{\mathrm{f}}(\mathrm{mm})$ & 63.5 & 67.5 & 53.9 & 66.1 & 57.9 & 55.4 \\
LY30 $^{\mathrm{g}}(\%)$ & 5.8 & 46.8 & 27.5 & 9.5 & 8.2 & 6.0 \\
LY60 $^{\mathrm{h}}(\%)$ & 22.9 & 54.0 & 45.5 & 19.8 & 10.1 & 10.2 \\
\hline
\end{tabular}

${ }^{a}$ APTT, activated partial thromboplastin time.

b $\mathrm{PT}$, prothrombin time.

${ }^{c} \mathrm{R}$, reaction time.

${ }^{\mathrm{d}} \mathrm{K}$, clot formation time.

${ }^{ } \alpha$, angle.

${ }^{\mathrm{f}}$ MA, maximal amplitude.

${ }^{g}$ LY30, lysis after $30 \mathrm{~min}$.

${ }^{\mathrm{h}}$ LY60, lysis after $60 \mathrm{~min}$.

recorded on the radiographs. However, this study does provide data to support normal VHS in red pandas that to our knowledge has not been reported previously.

Eosinophilia, thrombocytopenia, and neutrophilia are inconsistent findings in dogs infected with $A$. vasorum, depending on severity of the infection. ${ }^{5,21}$ Here, all hematologic parameters were within reference intervals, further supporting that this novel metastrongyloid nematode in the red panda has little clinical importance. Serum fructosamine was lower in the infected animals (288-319 $\mu \mathrm{mol} / \mathrm{L})$ compared with the controls (399-427 $\mu \mathrm{mol} / \mathrm{L})$. This is in concurrence with reported canine $A$. vasorum cases. ${ }^{20,21}$ Hyperglobulinemia has been reported quite frequently in dogs infected with $A$. vasorum ${ }^{5}$ but was not seen in this study. An increase in serum amylase was recorded in the group of infected animals. Amylase, however, is a very nonspecific biochemical parameter. In this respect, it cannot be excluded that these minor changes in serum biochemistry are related to different housing conditions because the infected and control animals came from different zoos. As a consequence, no conclusion can be made regarding whether the slightly elevated values recorded in the infected red pandas can be attributed to the infection with the novel metastrongyloid nematode.

Coagulopathies and clinical bleeding are commonly reported in canine $A$. vasorum cases. ${ }^{5,7,14}$ Here, no differences in measured APTT, PT, and fibrinogen were detected between the two groups. TEG is a diagnostic modality used to assess global hemostatic function. TEG measures clotting time, clotting kinetics, overall clot strength, and fibrinolytic activity. Neither TEG nor traditional coagulation parameters have been described in red pandas before this study; therefore, no reference values exist for this species. The assay was chosen because it has shown broad cross-species applicability, despite being activated with human TF. ${ }^{19}$ A few differences were detected between two of the infected animals and the controls (Table 4). $\mathrm{R}$ and $\mathrm{K}$ seemed to be slightly longer in the infected animals versus the controls. The difference was minor, and if it is clinically relevant, it may be explained by a mild consumption of coagulation factors in the infected animals. $\alpha$ and MA were the same for the two groups, indicating that there is no difference in clot kinetics or total clot strength in the healthy and diseased animals. MA correlates well to clinical signs of bleeding; thus, the results are as expected based on the clinical picture. The only substantial difference in TEG values was recorded in two of the infected animals (P2 and P3) that had an increased fibrinolytic activity with significantly higher lysis after 30 and 60 min (LY30 and LY60).

In summary, the clinical and paraclinical findings in the four infected red pandas were largely unremarkable, suggesting that infection with the newly discovered metastrongyloid nematode in the red pandas does not cause enough damage to affect the overall health of the animals. This conclusion is supported by 32 of 115 red pandas found to be infected with the parasite without apparent symptoms in a recent survey. ${ }^{1}$ A possible explanation is that this represents a red pandaspecific parasite imported with the animals and maintained within the zoo population rather than a parasite acquired in Europe zoos. Both $A$. vasorum and the novel parasite are diagnosed by detection of $\mathrm{L}_{1}$ larvae excreted in feces by the Baermann method. However, morphologic distinction between the $\mathrm{L}_{1}$ larvae of $A$. vasorum and the novel nematode is challenging. ${ }^{1}$ In this respect, diagnostic modalities such as polymerase chain reaction might be of importance to make the clear distinction between the two parasites. This might aid in distinguishing between infection with $A$. vasorum that is possibly lethal, and infection with the novel metastrongyloid that apparently causes little damage. Regarding potential treatment recommendations, it can be argued to treat all infected animals, thereby eliminating 
the risk of $A$. vasorum infections going untreated. However, this only makes sense if a safe and efficacious treatment against both parasites is available, which currently is not the case for red pandas. Another approach could be to only treat animals diagnosed as infected if they proceed to show clinical signs. Further research is needed to show which recommendations would be the best option.

Acknowledgments: The study was cofunded by the Department of Small Animal Clinical Sciences, Faculty of Health and Medical Sciences, University of Copenhagen and Copenhagen Zoo. We thank the staff at Aalborg Zoo and Gistrup Veterinary Clinic for help and for the use of facilities during examination of the four red pandas in the infected group. From Department of Small Animal Clinical Sciences, Faculty of Health and Medical Sciences, University of Copenhagen, we thank Anette Urbrand Martinsen for valuable technical support and assistance and Prof. Dorte Hald Nielsen for evaluation of thoracic radiographs.

\section{LITERATURE CITED}

1. Bertelsen, M. F., J. L. Willesen, F. MeylandSmith, R. Jefferies, E. R. Morgan, and J. Monrad. 2010. Diversity and prevalence of metastrongyloid nematodes infecting the red panda (Ailurus fulgens) in European zoos. Vet. Parasitol. 172: 299-304.

2. Boag, A. K.., C. R. Lamb, P. S. Chapman, and A. Boswood. 2004. Radiographic changes in 16 dogs infected with Angiostrongylus vasorum. Vet. Rec. 154: 426-430.

3. Bolt, G., J. Monrad, J. Koch, and A. L. Jensen. 1994. Canine angiostrongylosis-a review. Vet. Rec. 135: 447-452.

4. Buchanan, J. W., and J. Bucheler. 1995. Vertebral scale system to measure canine heart size in radiographs. J. Am. Vet. Med. Assoc. 206: 194-199.

5. Chapman, P. S., A. K. Boag, J. Guitian, and A. Boswood. 2004. Angiostrongylus vasorum infection in 23 dogs (1999-2002). J. Small Anim. Pract. 45: 435-440.

6. Choudhury, A. 2001. An overview of the status and conservation of the red panda Ailurus fulgens in India, with reference to its global status. Oryx 35: 250259.

7. Cury, M. C., W. S. Lima, M. P. Guimaraes, and M. G. Carvalho. 2002. Hematological and coagulation profiles in dogs experimentally infected with Angiostrongylus vasorum (Baillet, 1866). Vet. Parasitol. 104: 139-149.

8. Glatston, A. R. 2008. International Red Panda Studbook. Ailurus fulgens. Rotterdam Zoo, Rotterdam, The Netherlands.
9. Grøndahl, C., J. Monrad, H. H. Dietz, H. E. Jensen, M. V. Johansen, and C. Kapel. 2005. Angiostrongylosis in red panda (Ailurus fulgens fulgens). Erkrankungen der Zootiere. Proc. Int. Symp. Zoo Wild Anim. Dis. 42: 117-118.

10. International Species Information System (ISIS). 2002. Reference Values for Physiological Values in Captive Wildlife. ISIS, Apple Valley, Minnesota.

11. Jensen, H. E., H. H. Dietz, C. Grøndahl, C. Kapel, and J. Monrad. 2005. The pathology associated with Angiostrongylus vasorum infection in the red panda (Ailurus fulgens fulgens). Proceedings of the 23rd Meeting of the European Society of Veterinary Pathology, The European Society of Veterinary Pathology. Pp. 70-71.

12. Jones, M. L. 1989. A brief history of the red panda in captivity. In: Glatston, A. R. (ed.). Red Panda Biology. SPB Academic Publishing, The Hague, The Netherlands. Pp. 9-24.

13. Kjelgaard-Hansen, M., and A. L. Jensen. 2006. Is the inherent imprecision of manual leukocyte differential counts acceptable for quantitative purposes? Vet. Clin. Pathol. 35: 268-270.

14. Koch, J., and J. L. Willesen. 2009. Canine pulmonary angiostrongylosis-an update. Vet. J. 179: 348-359.

15. Patterson-Kane, J. C., L. M. Gibbons, R. Jefferies, E. R. Morgan, N. Wenzlow, and S. P. Redrobe. 2009. Pneumonia from Angiostrongylus vasorum infection in a red panda (Ailurus fulgens fulgens). J. Vet. Diagn. Invest. 21: 270-273.

16. Roberts, M. S., and J. L. Gittleman. 1984. Ailurus fulgens. Mam. Species 222: 1-8.

17. Wei, F. W., Z. J. Feng, Z. W. Wang, and J. C. Hu. 1999. Current distribution, status and conservation of wild red pandas Ailurus fulgens in China. Biol. Conserv. 89: 285-291.

18. Wiinberg, B., A. L. Jensen, R. Rojkjaer, P. Johansson, M. Kjelgaard-Hansen, and A. T. Kristensen. 2005. Validation of human recombinant tissue factor-activated thromboelastography on citrated whole blood from clinically healthy dogs. Vet. Clin. Pathol. 34: 389-393.

19. Wiinberg, B., and A. T. Kristensen. 2010. Thromboelastography in veterinary medicine. Semin. Thromb. Hemost. 36: 747-756

20. Willesen, J. L., A. L. Jensen, A. T. Kristensen, M. Kjelgaard-Hansen, R. Jessen, and J. Koch. 2006. Serum fructosamine concentrations in 59 dogs naturally infected with Angiostrongylus vasorum. J. Vet. Med. A 53: 266-269.

21. Willesen, J. L., A. L. Jensen, A. T. Kristensen, and J. Koch. 2009. Haematological and biochemical changes in dogs naturally infected with Angiostrongylus vasorum before and after treatment. Vet. J. 180: 106111.

Received for publication 18 May 2011 